\title{
Molecular Image-Guided Theranostic and Personalized Medicine
}

\author{
Hong Zhang, ${ }^{1}$ Mei Tian, ${ }^{2}$ Carrio Ignasi, ${ }^{3}$ Zhen Cheng, ${ }^{4}$ Lie-Hang Shen, ${ }^{5}$ and David J. Yang ${ }^{2}$ \\ ${ }^{1}$ The Second Affiliated Hospital of Zhejiang University, Hangzhou, Zhejiang 310009, China \\ ${ }^{2}$ The University of Texas MD Anderson Cancer Center, Houston, TX 77030, USA \\ ${ }^{3}$ Nuclear Medicine Department, Hospital Sant Pau, 08025 Barcelona, Spain \\ ${ }^{4}$ Molecular Imaging Program at Stanford and Bio-X Program, Stanford University, Stanford, CA 94305, USA \\ ${ }^{5}$ Institute of Nuclear Energy Research, Taoyuan 32546, Taiwan
}

Correspondence should be addressed to David J. Yang, dyang@mdanderson.org

Received 27 April 2011; Accepted 27 April 2011

Copyright (C) 2011 Hong Zhang et al. This is an open access article distributed under the Creative Commons Attribution License, which permits unrestricted use, distribution, and reproduction in any medium, provided the original work is properly cited.

The growing use of molecular imaging helps to control and monitor dosage for increased safety and effectiveness. Molecular imaging agents become the scientific tools for moving a concept from bench work to clinic product development. This special issue provides original research and review of new trends in molecular imaging agent development.

Among clinic molecular imaging modalities, radionuclide imaging technique is the most sensitive and could provide target-specific information. The radiotracer could noninvasively assess disease treatment endpoints which used to rely almost exclusively on biopsies and histopathological assays.

L.-H. Shen et al. (Taiwan) reviewed molecular imaging in the diagnosis and management of CNS disorders. F.-L. Kong et al. (USA), N. Tsao et al. (USA), and C.-L. Ho et al. (Taiwan) report pharmacokinetics and dosimetry of molecular imaging compounds in oncology. M.-H. Liao et al. (Taiwan), X. Wang et al. (China), C. Wu et al. (China) and M. Tian et al. (USA) reported that molecular imaging techniques play a major role in the development of novel therapies since they measure target expressions (apoptosis, glycolysis) as well as function, pathway activities, and cell migration in the intact organism. Y.-F. Wang et al. and M.-Y. Chao et al. (Taiwan) described the kit probe for ${ }^{99 \mathrm{~m}} \mathrm{Tc}$-labeling which is crucial for centralized drug process. The kit probe may allow theranostic concept. A theranostic agent would be more accurate in the evaluation of patient response to treatment. The radiotracer could also be used for radionuclide therapy as reported by $\mathrm{C}$. Wang et al. (China). Hybrid molecular imaging modalities (PET/CT,
SPECT/CT) provide high-sensitivity functional and highresolution anatomical imaging which are important in design-personalized treatment. M.-C. Chen et al. (Taiwan) reported the value of using I-131 NP-59 SPECT/CT to diagnose patients with subclinical or atypical features of primary aldosteronism. To proof of concept for molecular biology findings using different imaging modalities (optical, sonography) were described by Z. Pu et al. (China).

In summary, molecular imaging enables the comprehensive characterization of therapeutic intervention and can be used in preclinical studies, pharmacokinetic studies, dosefinding studies, and proof-of-concept studies. Molecular imaging technologies promote the discovery and development of personalized medicine. 


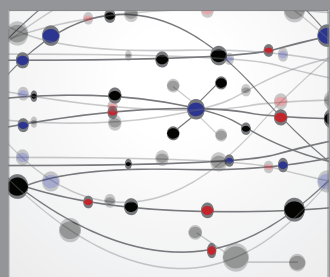

The Scientific World Journal
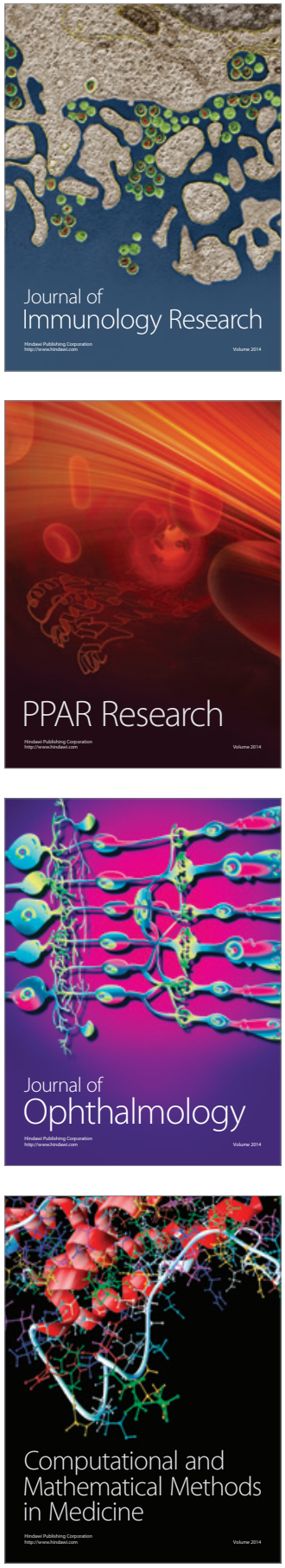

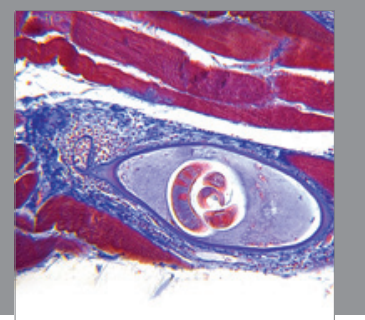

Gastroenterology

Research and Practice
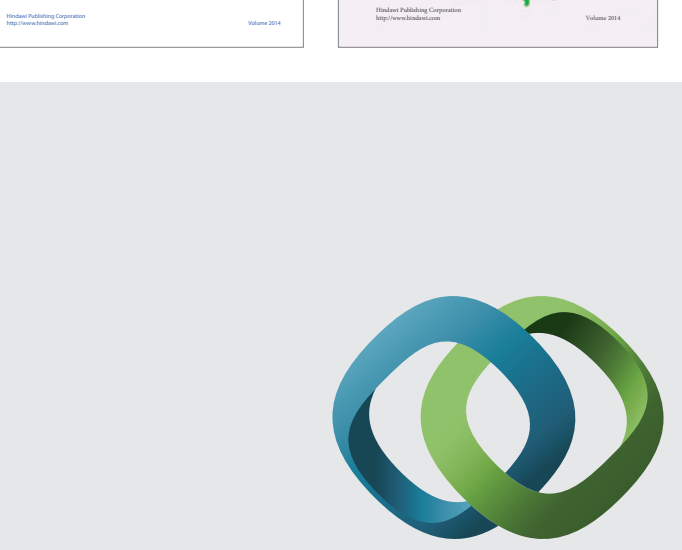

\section{Hindawi}

Submit your manuscripts at

http://www.hindawi.com
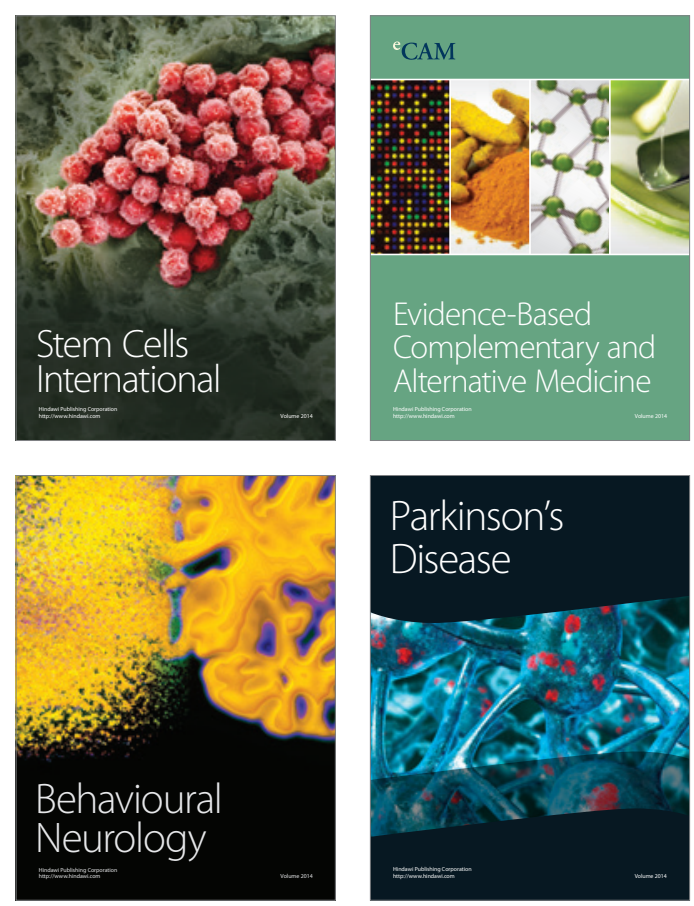

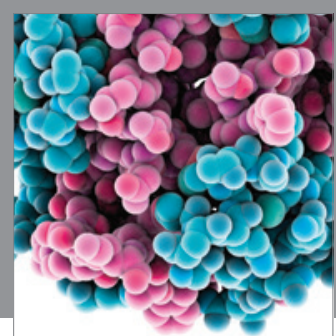

Journal of
Diabetes Research



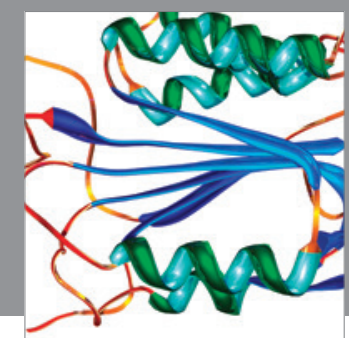

Disease Markers
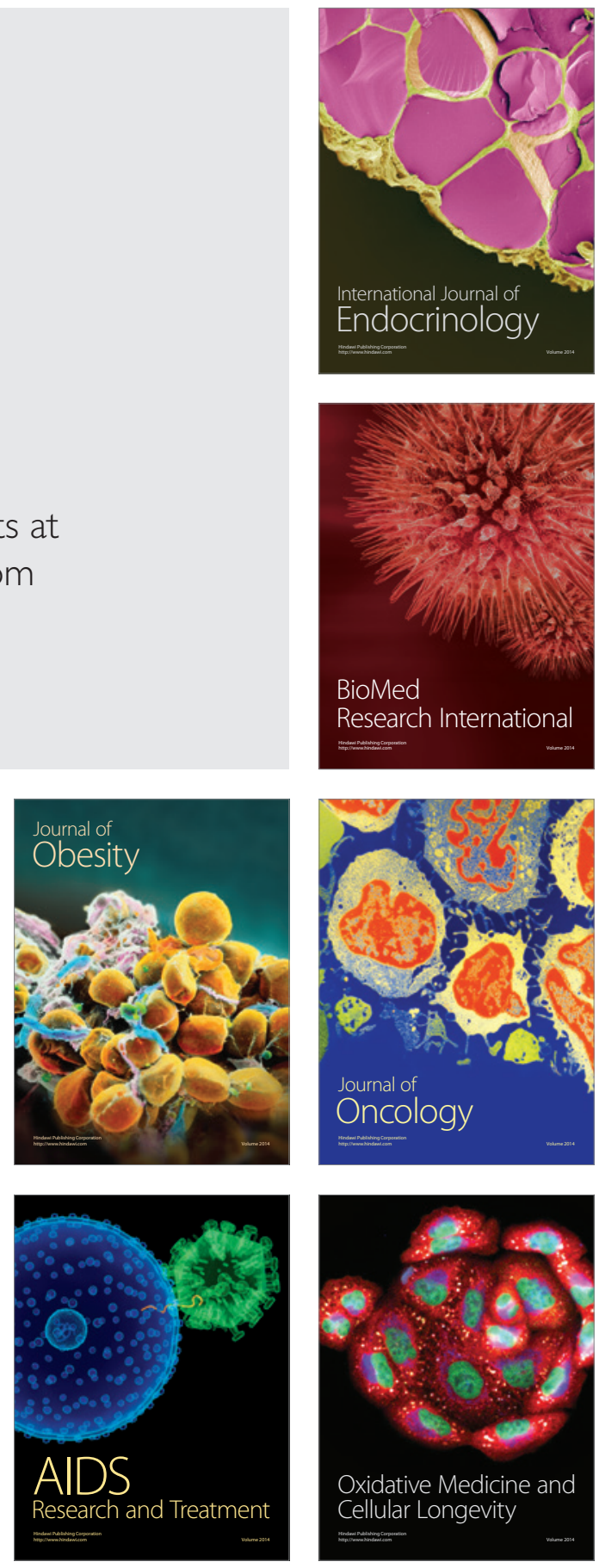